\title{
Conformal Patch Antenna Arrays Design for Onboard Ship Deployment Using Genetic Algorithms
}

\author{
Stelios A. Mitilineos, ${ }^{1}$ Symeon K. Symeonidis, ${ }^{1}$ Ioannis B. Mpatsis, ${ }^{2}$ Dimitrios Iliopoulos, ${ }^{1}$ \\ Georgios S. Kliros, ${ }^{3}$ Stylianos P. Savaidis, ${ }^{1}$ and Nikolaos A. Stathopoulos ${ }^{1}$ \\ ${ }^{1}$ Technologial Educational Institute of Piraeus, Department of Electronics, 250, Petrou Ralli kai Thivon Street, 12244 Aigaleo, Greece \\ ${ }^{2}$ Hellenic Air Force Academy, Dekeleia Air-Force Base, Dekeleia, Greece \\ ${ }^{3}$ Department of Aeronautical Sciences, Hellenic Air Force Academy, Dekeleia Air-Force Base, Dekeleia, Greece
}

Correspondence should be addressed to Stelios A. Mitilineos; smitil@gmail.com

Received 17 October 2012; Accepted 17 February 2013

Academic Editor: John Prousalidis

Copyright (c) 2013 Stelios A. Mitilineos et al. This is an open access article distributed under the Creative Commons Attribution License, which permits unrestricted use, distribution, and reproduction in any medium, provided the original work is properly cited.

Conformal antennas and antenna arrays (arrays) have become necessary for vehicular communications where a high degree of aerodynamic drag reduction is needed, like in avionics and ships. However, the necessity to conform to a predefined shape (e.g., of an aircraft's nose) directly affects antenna performance since it imposes strict constraints to the antenna array's shape, element spacing, relative signal phase, and so forth. Thereupon, it is necessary to investigate counterintuitive and arbitrary antenna shapes in order to compensate for these constraints. Since there does not exist any available theoretical frame for designing and developing arbitrary-shape antennas in a straightforward manner, we have developed a platform combining a genetic algorithm-based design, optimization suite, and an electromagnetic simulator for designing patch antennas with a shape that is not a priori known (the genetic algorithm optimizes the shape of the patch antenna). The proposed platform is further enhanced by the ability to design and optimize antenna arrays and is intended to be used for the design of a series of antennas including conformal antennas for shipping applications. The flexibility and performance of the proposed platform are demonstrated herein via the design of a highperformance GPS patch antenna.

\section{Introduction}

Conformal antennas and antenna arrays (arrays) inherit their name from the fact that they "conform" to the shape of a 2D yet not planar surface. More specifically, conformal antennas are flat curving antennas that follow or are embedded to an object of predefined shape, like that of an aircraft's nose. Conformal antennas and antenna arrays (arrays) have become necessary for vehicular communications (where a high degree of aerodynamic drag reduction is needed) due to their so-called "conformity" to arbitrary surface shape, like in avionics and high-performance ships or submarines.

Conformal antennas were developed in the 1980s in order to be integrated with the outer metallic layers of aircrafts, with the purpose of reducing the aerodynamic drag and improving aircraft speed, fuel consumption, and gas emissions. Conformal antennas gradually replaced conventional ones that project from the aircrafts' hull. As long as commercial applications are considered (including shipping), the technical limitations and constraints are similar to avionics, but the application of conformal antennas was until recently limited due to the high related costs of etching and integration.

However, in the recent years the reduction of these costs has turned conformal antennas to an attractive choice for civilian applications as well, from train antennas to car radio antennas in order to improve shape and aesthetics as well as increase vehicle performance, and to cellular base station antennas to save space and make antennas less visually intrusive [1].

Conformal antennas may also be used in maritime communications and shipping applications, to not only reduce aerodynamic or-in this case-"hydrodynamic" drag, but also offer the ability to integrate more antenna elements in the ship's hull, offering a very large surface for antenna 


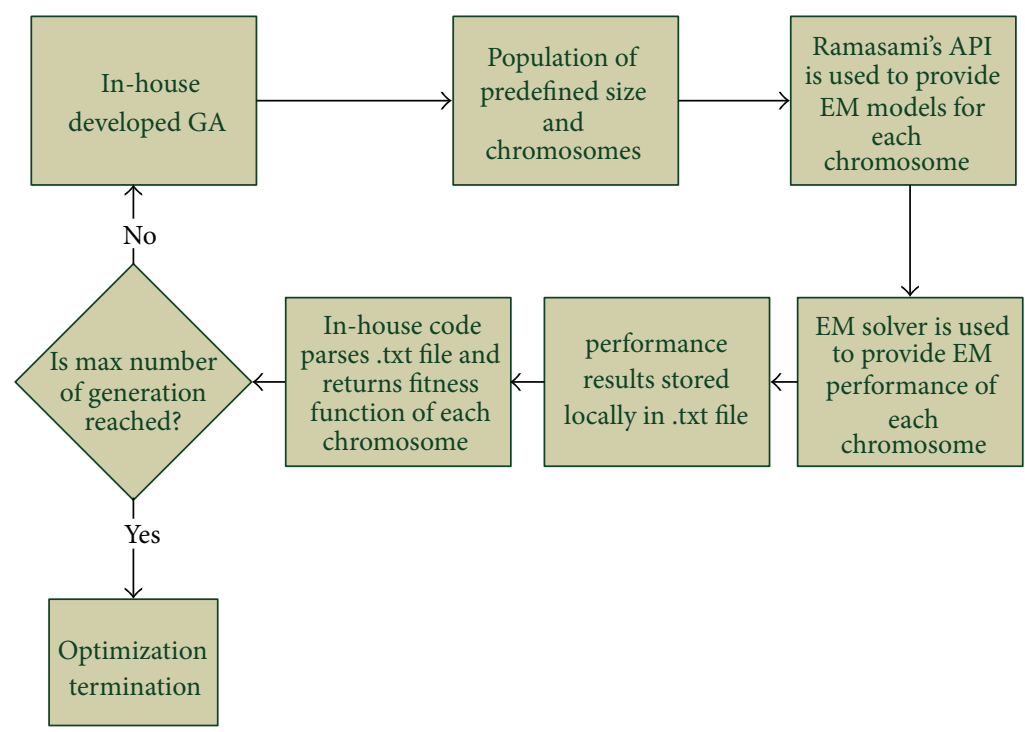

FIGURE 1: Flowchart of the proposed GA-based optimization platform for arbitrary-shape patch antennas.

deployment that could in turn yield to increased performance with limited overall antenna profile (volume, visually intrusiveness). Such an example of a GPS-receiving patch antenna is presented in this paper that demonstrates the abilities and performance of a proposed platform for arbitrary-shape flat antennas design and optimization. The choice of a GPSappropriate antenna has been made on the basis that GPS has become one of the top most important services necessary for a ship nowadays; GPS is used in ships not only to monitor its route and navigation, but also for safety and security. Other, more sophisticated GPS applications in shipping include synchronizing phasor measurement units for hybrid state estimators to provide estimates from SCADA control units on board, like electric power quality, ship performance monitoring, hull and machinery structural fatigue, and so forth [2], or speed determination with high accuracy, or even engine speed determination [3].

On top of the high related costs of integration, conformal antennas also suffer from severe constraints imposed on their design that arise due to the predefined and often counterproductive shape of the flat area that they need to conform with. This affects the performance of the antenna array, its shape, the elements spacing, the relative signal phase, and so forth. Thereupon, it is necessary to investigate counterintuitive and arbitrary antenna shapes in order to compensate for these constraints. Since there does not exist any available theoretical frame for designing and developing arbitrary-shape antennas in a straightforward manner, we have developed a combination of a genetic algorithm- (GA-) based design and an electromagnetic (EM) solver for designing patch antennas with a shape that is not a priori known (the genetic algorithm optimizes the shape of the patch antenna together with its other characteristics). The proposed GA is of high performance and proved in practice to deliver antenna patches of arbitrary shapes but of leveraged performance and low profile. Moreover, it is anticipated that the proposed platform is of generic use and may be readily deployed to other antenna and antenna arrays optimization problems as well. The flexibility and performance of the proposed platform are demonstrated herein via the design of a high-performance GPS patch antenna.

The rest of the paper is organized as follows. Section 2 illustrates an overall presentation of the proposed platform for the design of arbitrary-shape flat antennas, while Section 3 discusses in short the concept of patch antennas. Section 4 discusses the specifics of the genetic algorithm that has been employed for antenna optimization. Section 5 illustrates a GPS-suitable receiving antenna that was optimized using the proposed platform, and the paper concludes with Section 6.

\section{Integrated Platform for Antenna Design}

The proposed platform for antenna and antenna array design is based on combining an EM solver with an in-house developed GA solution. The proposed GA solution has been used in the past for the design and optimization of dipole antennas and antenna arrays [4-6] and is written with the MATLAB programming language.

In the proposed scheme, the GA is used in order to configure an initial population of 80 chromosomes (more information on GA terminology and literature is available in Section 4), that are then converted to valid EM models. Each model is simulated, and the results are locally stored in a .txt file. Then, our platform parses the .txt file and returns the fitness function of each chromosome to the GA. After the execution of the aforementioned steps for the initial population, our GA employs selection, mating, and mutation tactics to develop new generations up to a maximum of 100 generations. The proposed platform's internal structure is illustrated in Figure 1.

\section{Patch Antennas}

Microstrip patch antennas were first introduced during the second half of the twentieth century and are based on 


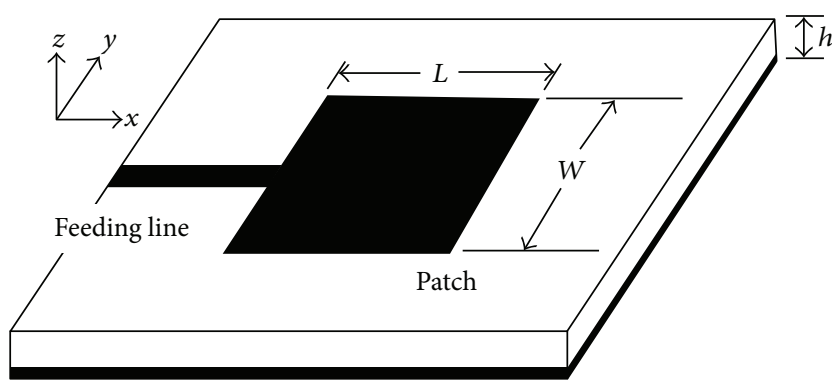

Ground plane

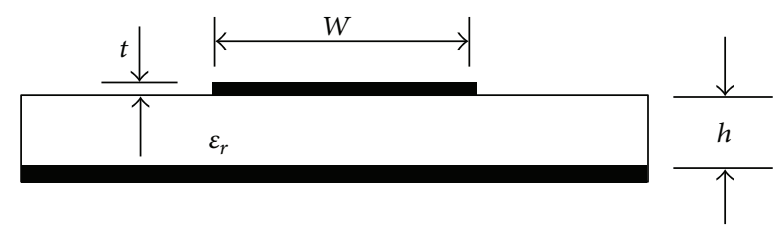

Figure 2: Patch antenna schematic example.

the observance that microstrips may radiate electromagnetic waves efficiently given certain limitations [7]. A patch antenna schematic example is depicted in Figure 2.

The width and length of the patch are illustrated in the top side of Figure 2, together with the feeding transmission line (the line is connected to the source). Also, there are a substrate of thickness $h$ and a ground plane. The substrate has a relative permittivity equal to $\varepsilon_{r}$, while the thickness of the patch is equal to $t$.

Due to inherent design limitations, patch antennas usually radiate most effectively towards the direction that is perpendicular to the substrate surface and opposite to the ground plane. Note that, by convention, there is a Cartesian coordinates system as the one depicted in the top side of Figure 2, so that the $z$-axis is always in line with the boresight of the patch.

Different patch layouts are proposed in the literature, yielding rectangular, circular, ring, or other complex patch layouts. Nevertheless, there are no analytic expressions for arbitrary-shape patches, like the one we propose herein; in such cases, one can only work with numerical electromagnetic solvers.

Furthermore, a patch antenna may be fed using either a microstrip or coaxial probes (see Figure 3), or even using sophisticated techniques of induced fields due to proximity to nearby transmission lines, and so forth $[7,8]$. The patch antenna proposed herein is using a coaxial probe feeding technique (like in Figure 3, bottom side) with a standard SMA connector and cables.

\section{Genetic Algorithm and Fitness Function Used}

GAs are a robust class of stochastic optimization algorithms, especially suited for nonlinear, nondifferential, multiobjective, and multidimensional optimization problems. They have been introduced in early 1960s, but only recently have they
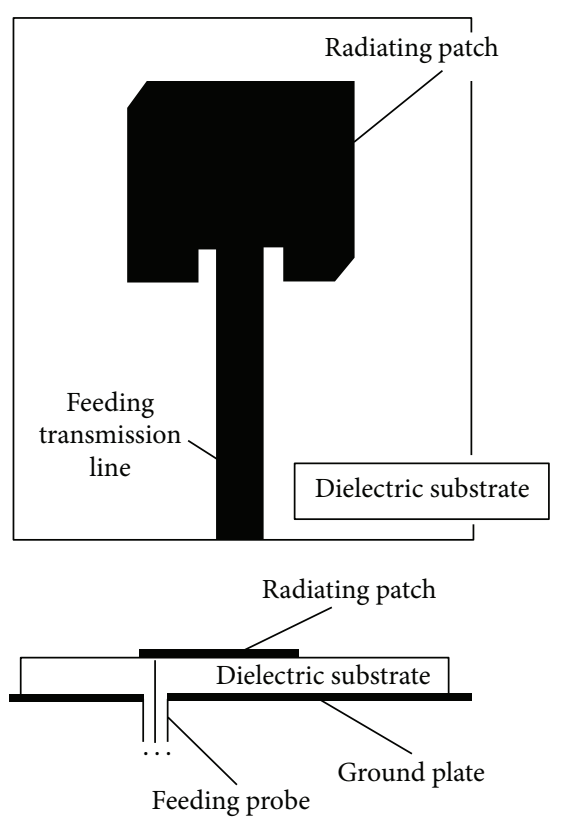

FIGURE 3: Various patch feeding techniques.

been widely used for electromagnetic optimization [9-11]. In GAs, the problem under search is properly parameterized, and a set of possible solution vectors or chromosomes is randomly generated. The elements of each vector or genes correspond to the problem parameters. The set of chromosomes is referred to as population or generation. A fitness function value is assigned to each chromosome, evaluating its suitability as a potential problem solution. Then, the stochastic search of the solution space is performed through a simulated genetic evolution, using selection, crossover, and mutation operators. New populations are generated, and the procedure is repeated, until a termination criterion is met.

GAs are considered to have certain advantages over other heuristic methods currently used, like the Particle Swarm Optimization (PSO) or the Simulated Annealing methods (SA). Even though it is difficult to establish a benchmark for heuristics algorithms [12], hands-on experience has shown that GAs converge faster and more often than other heuristic methods currently available, but one should always bear in mind that convergence heavily depends on the specific algorithm implementation [13].

The GA used herein has been in-house developed based on the work reported by Houck et al. [14] and has been used in the past in various dipole-antennas designs [4-6]. The GA is of the floating point type, while the functions of selection, crossover, and mutation are of roulette, simple or arithmetic, and uniform type, respectively. The population size is 80 , while the maximum number of generations has been set up to 100 .

The arbitrary patch of the antenna is optimized as follows: at first, one chromosome gene is designated to correspond to the patch's width and one more to the patch's length (see Figure 2). The genes' boundaries are subject to intuitive decision of the designer (for a $1.5 \mathrm{GHz}$ patch we set them 


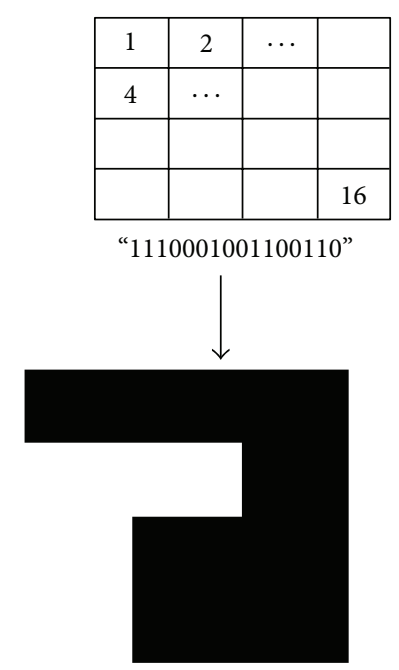

FIgURE 4: Patch antenna schematic example.

up as between $2 \mathrm{~cm}$ and $12 \mathrm{~cm}$ ). Then, the patch surface is split into 100 tiny rectangles, each of which has dimensions equal to $W / 10 \times L / 10$. Then, another 100 genes are assigned with a binary value of either 0 or 1 , with " 0 " corresponding to "no metal" and " 1 " corresponding to "metal." This means that if the GA assigns the value of " 0 " to a gene, then at the respective tiny rectangle there will be no metallic patch surface (and vice versa in the case of "1"). Thus, a patch of arbitrary shape is generated; by "arbitrary" we refer to both its dimensions and its specific shape since its surface varies according to the respective chromosome. For example, consider in Figure 4 the case of a patch with 16 (instead of 100) tiny rectangles. In the case where the chromosome had the value of " 1110001001100110 " assigned to the "metal or no metal" genes, then the patch shape would look like the one in the bottom of Figure 4.

Finally, a GA's performance strongly depends on the design of its fitness function. The fitness function of the proposed GA is developed as follows: after a patch model is generated by the GA's chromosome, it is passed to the EM solver according to Figure 1. The EM solver then outputs a.txt file that includes the gain, radiation pattern, and reflection coefficient of the patch. The fitness function takes into account that the maximum gain of the patch must be the highest possible; thereupon it first calculates an error value using

$$
e_{1}=\left[\frac{(5-\text { MaxGain })}{5}\right]^{2}
$$

where MaxGain is the maximum gain of the patch. The value of " 5 " is selected since the majority of commercially available patch antennas exhibits a gain of around $4 \mathrm{dBi}$; thus with this selection our patch is forced to outmatch this limit getting close to $5 \mathrm{dBi}$.
Then, the fitness function takes into account that the reflection coefficient, $S_{11}$, must be less than $-10 \mathrm{~dB}$; thereupon it calculates another error value as in

$$
e_{2}=\left[\frac{\left(10-S_{11}\right)}{10}\right]^{2} \text {. }
$$

Moreover, the fitness function also takes into account the variance of the patch's horizontal gain since it needs to be kept as low as possible for uniform radiation. Thus, the fitness function also calculates a third error term as in

$$
e_{3}=\sum_{\phi=0^{\circ}}^{\phi=360^{\circ}}\left(\text { MeanGain }_{\mathrm{phi}}-\operatorname{Gain}_{\mathrm{phi}}(\phi)\right)^{2}
$$

where MeanGain $_{\text {phi }}$ is the mean horizontal over $\phi$ for $\theta=20^{\circ}$, and $\operatorname{Gain}_{\mathrm{phi}}(\phi)$ is the horizontal gain versus $\phi$ for $\theta=20^{\circ}$.

Finally, the cumulative error is calculated by

$$
A=w_{1} \cdot e_{1}+w_{2} \cdot e_{2}+w_{3} \cdot e_{3},
$$

where $w_{1}$ is the weight of the error $e_{i}$, and the fitness function value is calculated by

$$
\text { Fitness }=\frac{1}{1+\sqrt{A}} .
$$

The specific formula for (5) is heuristic and implies that a "good" chromosome with a low cumulative error will correspond to a fitness function value tending to unity, while a "bad" chromosome with a large cumulative error will correspond to a fitness function value tending to zero.

\section{Design of a Flat Patch Antenna for GPS Applications}

Various runs of the proposed GA optimization platform have been executed with the purpose of designing a patch antenna of arbitrary shape suitable for applications around $1.5 \mathrm{GHz}$, like a GPS receiver. The substrate of the patch antenna should be of the FR4 type, with a substrate thickness of $1.6 \mathrm{~mm}$ and a dielectric constant equal to $\varepsilon_{r}=4.6$. Furthermore, the patch antenna should be fed via standardized SMA cables and connectors that are also integrated in the simulation, design, and optimization of the patch. Further specifications of the antenna include a maximum gain as high as possible at $\theta=0^{\circ}$ (referring to Figure 2 and with a specific target of a gain larger than $4 \mathrm{dBi}$, since this is a rather usual figure in most commercial patch elements), a reflection coefficient as low as possible (with a specific target of $-10 \mathrm{~dB}$, since this is considered to be the rule of thumb for RF and microwave components), and a bandwidth as large as possible.

After multiple runs it was decided that the various weights of the fitness function should be assigned, so as the final form of the latter should be

$$
A=4 \cdot e_{1}+2 \cdot e_{2}+1.8 \cdot e_{3} .
$$

With this adjustment, the GA was again executed multiple times, and the most promising patch antenna for GPS applications is presented herein. 


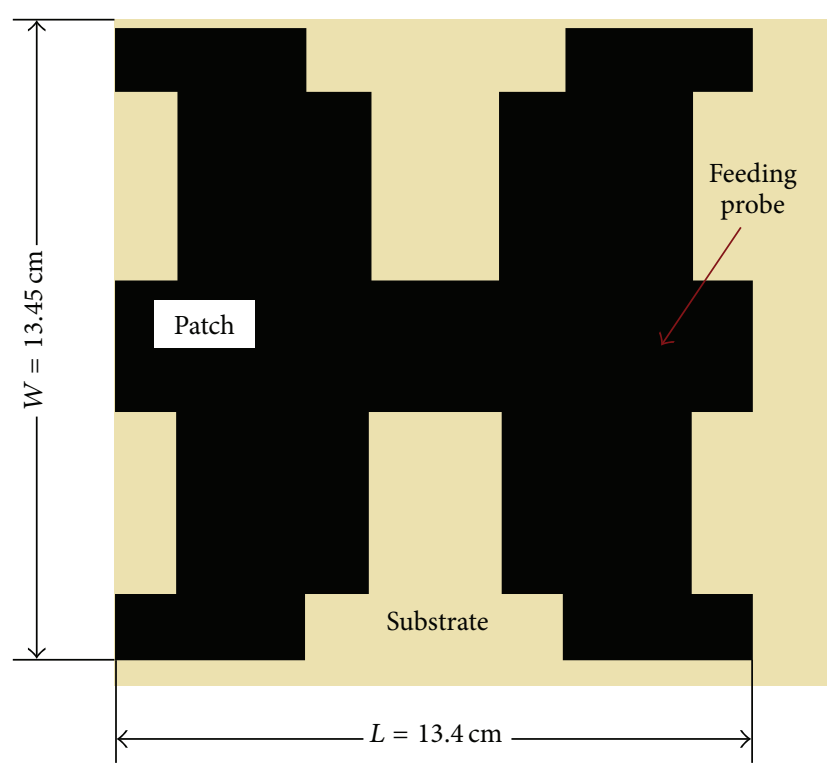

FIGURE 5: Layout of the optimized patch antenna (top view).

TABLE 1: Technical characteristics and performance results of the optimized patch antenna.

\begin{tabular}{lc}
\hline Central frequency $(\mathrm{GHz})$ & 1.5 \\
Total maximum gain (e- and H-plane) at central & 5 \\
frequency $(\mathrm{dBi})$ & -14 \\
Reflection coefficient at central frequency $(\mathrm{dB})$ & 0.3 \\
Standard deviation of horizontal gain for $\theta=20^{\circ}(\mathrm{dB})$ & 50 \\
\hline
\end{tabular}

The layout of the patch antenna is illustrated in Figure 5, while a summary of its performance results is tabulated in Table 1. It is considered that the optimized patch antenna exhibits superior gain $(5 \mathrm{dBi})$ compared to the literature and commercially available elements, with a low profile and easily converted to a conformal antenna. It is worthwhile noting that similar performance is usually available from antennas that are of significantly larger dimensions.

\section{Conclusions}

Using arbitrary-shape antennas may be a significant aid in developing high-performance antennas and arrays under strict constraints. Conformal antennas are a priori considered as a type of antennas that need to comply with such strict constraints and at the same time are of high importance for avionics and marine communications. With the proposed platform we were able to design a GPS antenna of low profile and high performance. Future work will include the design of planar antennas that will be etched at the outer metallic layers of ships and aircrafts using our design platform.

\section{References}

[1] L. Josefsson and P. Persson, Conformal Array Antenna Theory and Design, IEEE Press and John Wiley \& Sons, Hoboken, NJ, USA, 2006.

[2] A. G. Phadke and J. S. Thorp, Synchronized Phasor Measurements and Their Applications, Springer Science and Business Media, Springer, 2008.

[3] Z. S. Filipi and D. N. Assanis, "A nonlinear, transient, singlecylinder diesel engine simulation for predictions of instantaneous engine speed and torque," Journal of Engineering for Gas Turbines and Power, vol. 123, no. 4, pp. 951-959, 2001.

[4] S. A. Mitilineos, K. S. Mougiakos, and S. C. A. Thomopoulos, "Design and optimization of ESPAR antennas via impedance measurements and a genetic algorithm (antenna designer's notebook)," IEEE Antennas and Propagation Magazine, vol. 51, no. 2, pp. 118-123, 2009.

[5] S. A. Mitilineos and C. N. Capsalis, "A new, low-cost, switched beam and fully adaptive antenna array for $2.4 \mathrm{GHz}$ ISM applications," IEEE Transactions on Antennas and Propagation, vol. 55, no. 9, pp. 2502-2508, 2007.

[6] S. A. Mitilineos, P. I. Papakanellos, and C. N. Capsalis, "Compensation for elements discrepancies in array development using genetic algorithms," in Proceedings of the European Microwave Association, vol. 2, pp. 269-273, September 2006.

[7] N. A. Stathopoulos, S. P. Savaidis, and S. A. Mitilineos, "RF measurements and characterization of conductive textile materials," in Electronics and Computing in Textiles, S. Vassiliadis, Ed., Ventus Publishing ApS, 2012.

[8] C. A. Balanis, Antenna Theory: Analysis and Design, John Wiley \& Sons, Hoboken, NJ, USA, 2005.

[9] R. L. Haupt, "Introduction to genetic algorithms for electromagnetics," IEEE Antennas and Propagation Magazine, vol. 37, no. 2, pp. 7-15, 1995.

[10] J. M. Johnson and Y. Rahmat-Samii, "Genetic algorithms in engineering electromagnetics," IEEE Antennas and Propagation Magazine, vol. 39, no. 4, pp. 7-21, 1997.

[11] Y. R. Samii and E. Michielssen, Electromagnetic Optimization by Genetic Algorithms, John Wiley \& Sons, 1999.

[12] A. Stefek, "Benchmarking of heuristic optimization methods," in Proceedings of the 14th MECHATRONIKA International Symposium, Trencianske Teplice, Slovakia, June 2011.

[13] S. A. Mitilineos, Mitigation of Multipath Fading Using Smart Antenas [Ph.D. thesis], NTUA Press, Athens, Greece, October 2006.

[14] C. R. Houck, J. A. Joines, and M. G. Kay, "A genetic algorithm for function optimization: a MATLAB implementation," Tech. Rep. NCSU-IE 95-09, 1995. 

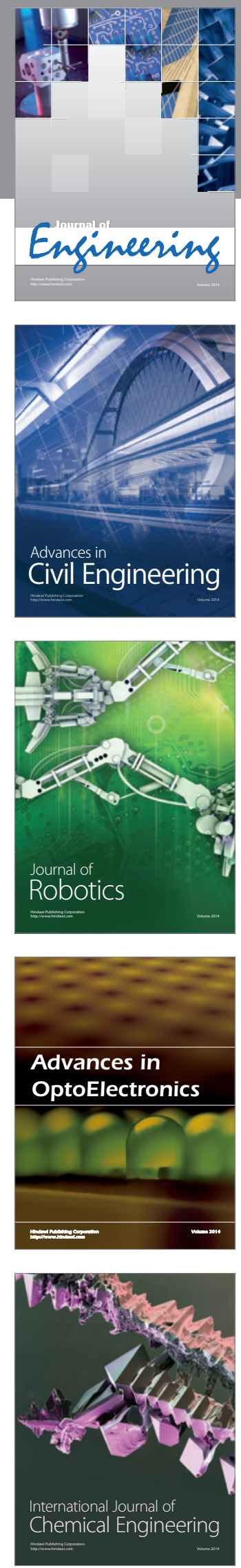

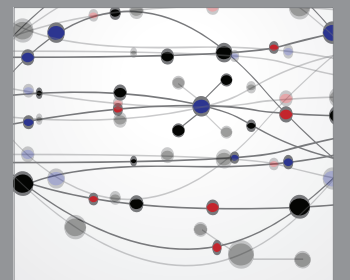

The Scientific World Journal
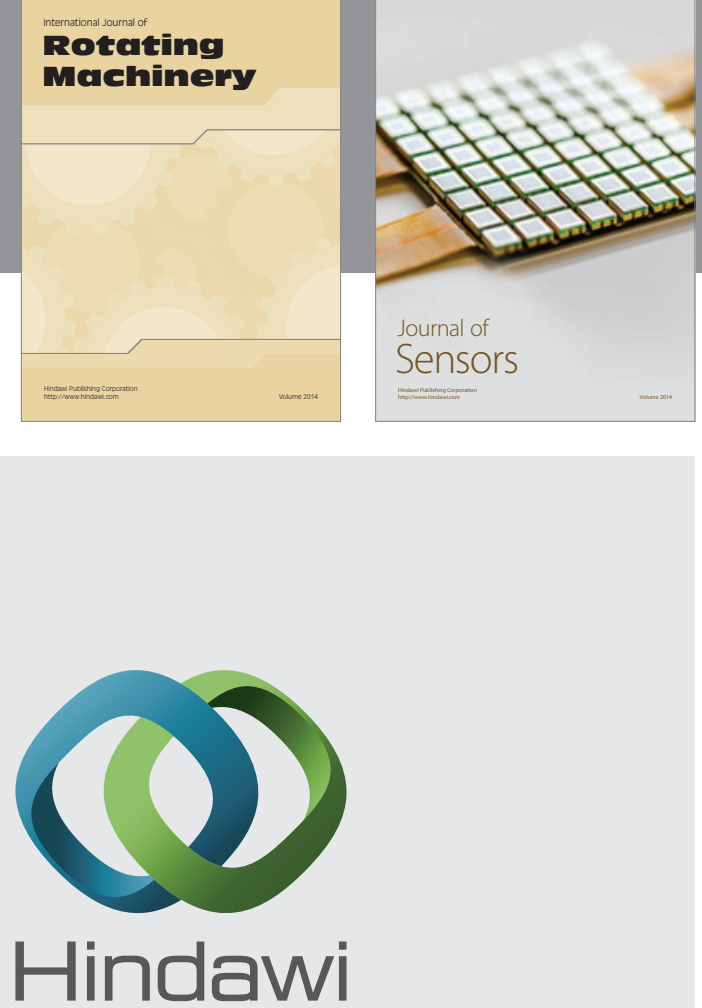

Submit your manuscripts at http://www.hindawi.com
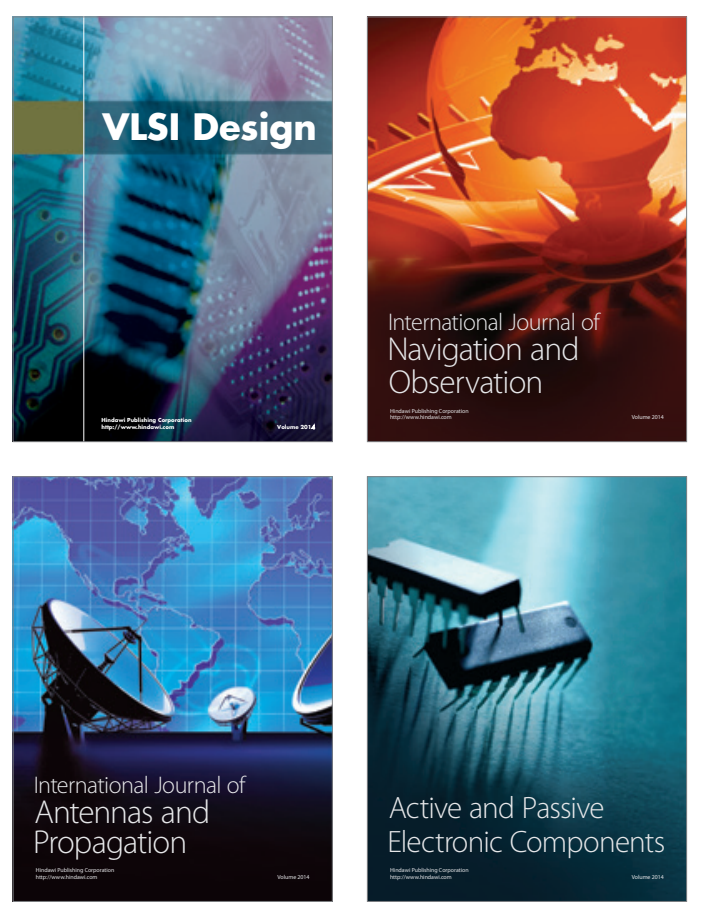
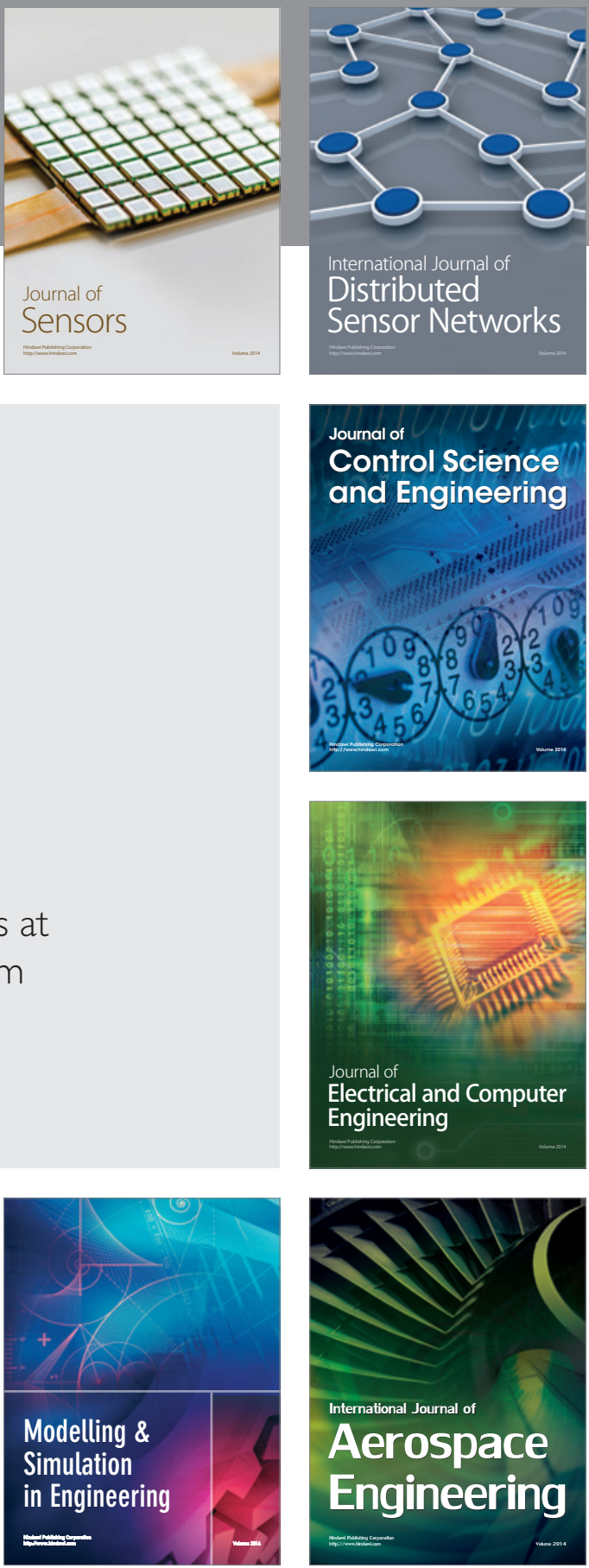

Journal of

Control Science

and Engineering
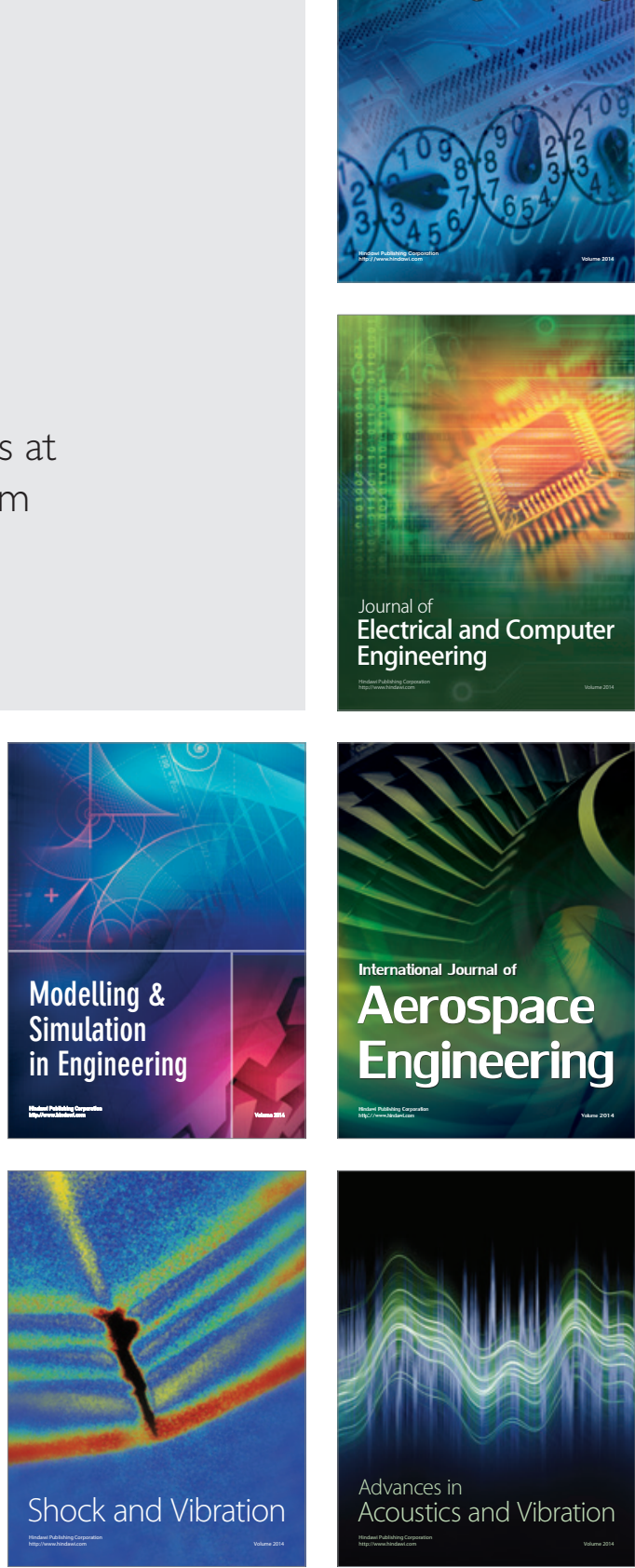